
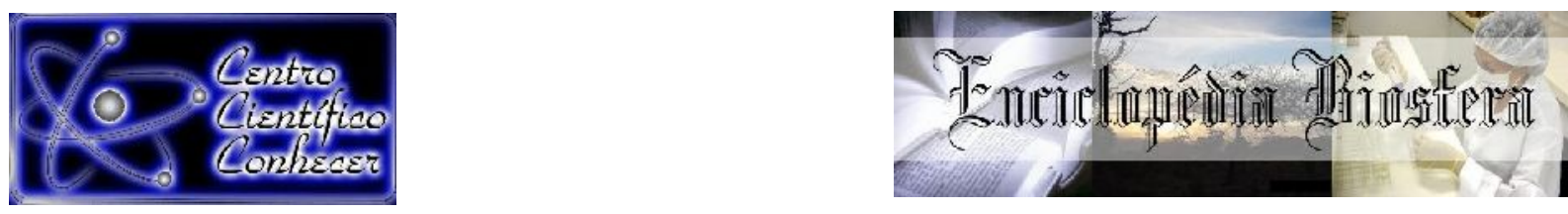

\title{
CRESCIMENTO DO ANADOR EM DIFERENTES TIPOS DE SUBSTRATOS
}

\author{
Aladje Té ${ }^{1}$, Ciro de Miranda Pinto ${ }^{2}$, Olienaide Ribeiro de Oliveira Pinto ${ }^{3}$, João \\ Gutemberg Leite Moraes ${ }^{4}$, Juan Carlos Alvarado Alcócer ${ }^{5}$.
}

\begin{abstract}
${ }^{1}$ Bacharel em Agronomia, Universidade da Integração Internacional da Lusofonia Afro-Brasileira (UNILAB), Redenção, Ceará, Brasil.

${ }^{2}$ Doutor, Professor do Instituto de Desenvolvimento Rural (IDR), Universidade da Integração Internacional da Lusofonia Afro-Brasileira (UNILAB), Redenção, Ceará, Brasil.

${ }^{3}$ Doutora, Bolsista do Programa Nacional de Pós-Doutorados (PNPD) /CAPES, Mestrado Acadêmico em Sociobiodiversidade e Tecnologias Sustentáveis (MASTS), Universidade da Integração Internacional da Lusofonia Afro-Brasileira (UNILAB), Redenção, Ceará, Brasil. Email: agron.olienaide@gmail.com

${ }^{4}$ Doutor, Professor do Instituto de Desenvolvimento Rural (IDR), Universidade da Integração Internacional da Lusofonia Afro-Brasileira (UNILAB), Redenção, Ceará, Brasil.

${ }^{5}$ Doutor, Professor do Mestrado Acadêmico em Sociobiodiversidade e Tecnologias Sustentáveis (MASTS), Universidade da Integração Internacional da Lusofonia Afro-Brasileira (UNILAB), Redenção, Ceará, Brasil.
\end{abstract}

\section{Recebido em: 15/08/2021 - Aprovado em: 15/09/2021 - Publicado em: 30/09/2021 DOI: $10.18677 /$ EnciBio_2021C13 \\ trabalho licenciado sob licença Creative Commons Attribution-NonCommercial-NoDerivatives 4.0 International License.}

\begin{abstract}
RESUMO
O anador (Justicia pectoralis Jacq) é uma planta medicinal com propriedades antiinflamatórias, podendo ser usado na forma de decocção, infusão, sumo e xarope. Essa planta é bastante rustica, desenvolvendo em solos de vários tipos, e quando fertilizada com adubos orgânicos ou químicos, incrementa o número de folhas e ramos, partes empregadas na medicina popular. Este trabalho teve como objetivo investigar a interferência dos diferentes tipos de substratos e definir aquele que proporcione maior crescimento do anador. O experimento foi conduzido na Universidade da Integração Internacional da Lusofonia Afro-Brasileira (UNILAB), Campus das Auroras, com seguintes tratamentos: $T_{1}$ : testemunha (solo); $T_{2}$ : solo + esterco ovino ( $\left.3: 1\right) ; T_{3}$ : solo + cama de frango (3:1); $T_{4}$ : solo + esterco ovino + cama de frango $(2: 1: 1)$ e por último $T_{5}$ : solo + cama de frango + esterco ovino + bagana de carnaúba (1:1:1:1). O delineamento estatístico utilizado no experimento foi o inteiramente casualizado com cinco tratamentos e 4 repetições, sendo cada repetição composta por quatro sacos de mudas. Foi observado que, as variáveis número de folha (NF), número de ramos (NR), altura de plantas (ALT), matéria seca de ramos (MSR), matéria seca da parte área (MSPA), matéria seca total (MST) e a relação MSR/MSPA deram significativos pelo teste de $\mathrm{F}$ ao nível de $1 \%$ de probabilidade. A propagação do anador é recomendada nos substratos solo + cama de frango, solo + esterco ovino e solo + bagana de carnaúba.
\end{abstract}

PALAVRAS-CHAVE: Fertilização orgânica, Justicia pectoralis Jacq, Planta medicinal. 


\title{
GROWTH OF THE ANADOR IN DIFFERENT SUBSTRATES
}

\begin{abstract}
The anador (Justicia pectoralis Jacq) is a medicinal plant with anti-inflammatory properties and can be used as a decoction, infusion, juice and syrup. This plant is quite rustic, developing in soils of various types, and when fertilized with organic or chemical fertilizers, it increases the number of leaves and branches, parts used in folk medicine. This work aimed to investigate the interference of different types of substrates and define the one that provides greater growth of the anador. The experiment was conducted at the University of International Integration of Afro-Brazilian Lusofonia (UNILAB), Campus das Auroras, with the following treatments: T1: control (soil); T2: soil + sheep manure (3:1); T3: soil + chicken litter (3:1); T4: soil + sheep manure + chicken litter (2:1:1) and finally T5: soil + chicken litter + sheep manure + carnauba bagasse $(1: 1: 1: 1)$. The statistical design used in the experiment was completely randomized with five treatments and four repetitions, each repetition being composed of four bags of seedlings. It was observed that the variables leaf number (NF), number of branches (NR), plant height (ALT), branch dry matter (MSR), part area dry matter (MSPA), total dry matter (MST) and the MSR/MSPA ratio were significant by the $F$ test at the $1 \%$ probability level. The propagation of the swimmer is recommended in soil + chicken litter, soil + sheep manure and soil + carnauba bagasse substrates.
\end{abstract}

KEYWORDS: Justicia pectoralis Jacq, Medicinal plants, Organic fertilization.

\section{INTRODUÇÃO}

O emprego das plantas medicinais para fins terapêuticos é uma prática antiga como forma de cura para diversas doenças da humanidade, apresentando importância social, cultural e econômica, trazendo além de novos conhecimentos, a utilização dessas espécies na saúde tem aumentado com avanço da experimentação e farmacêutica (PRADO et al., 2018; SILVA et al., 2021).

$O$ interesse no tradicional uso terapêutico de produtos derivados de plantas medicinais proporciona avanços de pesquisas científicas reconhecendo a eficácia e segurança dos efeitos de compostos vegetais existentes. Dentre diversas plantas medicinais aproveitadas para esse fim, o anador (Justicia pectoralis Jacq.) é conhecida popularmente no Nordeste como chambá, sendo amplamente utilizado em preparações caseiras para o tratamento da tosse, bronquite e asma (LEAL et al., 2017).

$\mathrm{O}$ anador é uma planta medicinal conhecida popularmente como: chachambá, trevo-do-pará e trevo-cumaru, pertence à família Acanthaceae, tendo como características: perene, subereta e pode chegar a $40 \mathrm{~cm}$ de altura, folhas simples, membranáceas, estreitas e longas podendo variar entre 3 a $10 \mathrm{~cm}$ de comprimento, apresenta múltiplos usos medicinais, e as partes dessa planta usadas são: planta inteira, folhas, caule, ramos e flores (LORENZI; MATOS, 2008, CARVALHO et al., 2021).

A extinção e a necessidade maior de uso de plantas medicinais na atualidade fazem com que, o seu plantio se torne fundamental para a sua preservação e conservação (VIEIRA et al., 2016). De acordo Baracuhy et al. (2016) o cultivo de plantas medicinais deve merecer um grande cuidado, sendo que, é uma etapa importante na obtenção do produto final (medicamento) e que se não for realizada de forma adequada 
poderá interferir na produção e qualidade de um medicamento fitoterápico, tanto do ponto de vista qualitativo quanto quantitativo.

Segundo Lameira e Pinto (2008) a reprodução das plantas medicinais são realizadas por sementes, além disso, podem ser disseminadas através de estacas que são partes vegetativas de plantas e, nesse caso é utilizado sacos plásticos, bandejas e vasos já preparados a fim de receber as estacas.

A propagação vegetativa é uma das formas mais utilizadas para a produção de mudas de plantas medicinais (HARTMANN et al., 2018). A partir de órgãos vegetativos (ramos, folhas, caules e até mesmo raízes), são obtidas estacas, portanto, a nova planta será originada a partir de seu enraizamento (GOELZER et al., 2019).

A adubação de plantas medicinais, de acordo com Lameira e Pinto (2008) pode ser realizada preferencialmente com adubação orgânica utilizando esterco de curral curtido e, também, cama de frango evitando composto orgânico produzido a partir de lixos orgânicos, porque estes podem conter metais pesados, pois podem prejudicar a qualidade das plantas. Combinar diferentes substratos contribui para ampliação das características dos substratos, boa retenção de água, aeração e proporciona maior crescimento e desenvolvimento das plantas (MASIERO et al., 2019). Assim, este trabalho teve como objetivo investigar a interferência dos diferentes tipos de substratos e definir aquele que proporcione maior crescimento do anador.

\section{MATERIAL E MÉTODOS \\ Localização de área experimental e origem do material vegetal}

O experimento foi conduzido na Universidade da Integração Internacional da Lusofonia Afro-Brasileira (UNILAB) Campus das Auroras, situado no município de Redenção, Ceará, nas coordenadas geográficas: latitude (S) $4^{\circ} 13^{\prime} 33^{\prime \prime}$ e longitude (WGr) 38 43' 50" Nordeste com 88,8 m de altitude. O clima de Redenção é classificado como Tropical Quente Úmido, Tropical Quente Sub-úmido e Tropical Quente Semiárido Brando, a pluviosidade $1.062,0 \mathrm{~mm}$, temperatura média apresenta variação de $26^{\circ} \mathrm{C}$ a $28^{\circ} \mathrm{C}$ (IPECE, 2017a).

O material propagativo utilizado no experimento foi os ramos da planta do anador, que foram coletados na capital do estado do Ceará, Fortaleza, tendo coordenadas geográficas: latitude (S) 3 43'02" e longitude (WGr) $38^{\circ} 32^{\prime}$ '35" Nordeste (IPECE, 2017b).

\section{Delineamento Experimental}

O delineamento estatístico utilizado foi inteiramente casualizado com 5 tratamentos e 4 repetições compostas de 4 sacos por cada, totalizando 80 sacos. Os tratamentos foram: $\mathrm{T}_{1}$ : testemunha (solo); $\mathrm{T}_{2}$ : solo + esterco ovino (3:1); $\mathrm{T}_{3}$ : solo + cama de frango (3:1); $\mathrm{T}_{4}$ : solo + esterco ovino + cama de frango (2:1:1) e por último $\mathrm{T}_{5}$ : solo + cama de frango + esterco ovino + bagana de carnaúba (1:1:1:1).

Para condução do experimento foram preparados sacos plásticos de tamanho $20 \times 30 \mathrm{~cm}$ preenchidos com diferentes tipos de substratos. A propagação do anador ocorreu de forma vegetativa, utilizaram-se ramos de $10 \mathrm{~cm}$ de comprimento. Os ramos foram cultivados $1 / 3$ do comprimento. A irrigação foi feita manualmente e diariamente. A implantação do experimento ocorreu no dia 15 de maio do ano 2019. No dia 12 de outubro foram realizadas as avaliações de números de folhas (NF), números de ramos (NR) e Altura de plantas (AP). Para a variável AP. A colheita das plantas para secagem ENCICLOPÉDIA BIOSFERA, Centro Científico Conhecer - Jandaia-GO, v.18 n.37; p. 149 
e obtenção da matéria da parte aérea (MSPA) e raiz (MSR) no dia 14 de outubro, essa secagem na estufa no laboratório de Sementes da UNILAB. O experimento teve duração de 153 dias, do plantio a colheita.

\section{Variáveis Analisadas}

As variáveis analisadas foram: números de folhas (NF), números de ramos (NR) e Altura de plantas (AP). Para a variável AP, utilizou-se uma régua graduada de $50 \mathrm{~cm}$. O material vegetal foi acondicionado sacos de papel de $30 \times 12 \mathrm{~cm}$ comprimento. As variáveis estudadas foram matéria seca de raiz (MSR), matéria seca de parte área (MSPA), matéria seca total (MST). Esse material vegetal foi submetido à secagem no Laboratório de Sementes da UNILAB em estufa com a temperatura de $56{ }^{\circ} \mathrm{C}$ durante 24 horas, sendo depois pesado em balança de precisão. Com os dados MSR, MSPA e MST, estabeleceu-se a relação alométrica de MSR/MSPA.

\section{Análises estatísticas}

Os dados foram submetidos à análise de variância pelo teste $\mathrm{F}$ ao nível $1 \%$ ou $5 \%$ de probabilidade, já as médias dos tratamentos foram comparadas pelo teste Scott e Knott ao nível de $5 \%$ de probabilidade auxilio do software estatístico AgroEstat (BARBOSA; MALDONADO JUNIOR, 2015).

\section{RESULTADOS E DISCUSSÃO}

Durante o período de avaliação não foi verificado nenhum ataque de pragas e doenças nas plantas de anador. Na Tabela 1, de acordo com análise de variância, houve diferença significativa dos substratos em função das variáveis: número de folha $\mathrm{NF}(p<0,0001)$, número de ramos - NR $(p=0,0013)$, altura de plantas - ALT $(p<0,0001)$ e matéria seca de ramos - MSR $(p=0,0009)$, todos deram significativos pelo teste de $F$ ao nível de $1 \%$ de probabilidade.

TABELA 1. Resumo da análise variância e teste de Scott e Knott do número de folhas (NF), número de ramos (NR), altura de plantas (Alt. em $\mathrm{cm}$ ), matéria seca de raiz (MSR) em diferentes substratos, Redenção, Ceará, Brasil, 2019.

\begin{tabular}{|c|c|c|c|c|}
\hline \multirow[b]{2}{*}{ Fonte de variação } & \multicolumn{4}{|c|}{ Quadrado Médio } \\
\hline & NF & NR & Alt & MSR \\
\hline Tratamentos & $\begin{array}{l}728,075^{* *} \\
(p<0,0001)\end{array}$ & $\begin{array}{c}2,875^{* *} \\
(p=0,0013)\end{array}$ & $\begin{array}{c}81,120^{* *} \\
(p<0,0001)\end{array}$ & $\begin{array}{c}0,558^{* *} \\
(p=0,0009)\end{array}$ \\
\hline Resíduo & 23,966 & 0,366 & 0,903 & 0,066 \\
\hline Substratos & \multicolumn{4}{|c|}{ Teste de Scott e Knott } \\
\hline $\mathrm{T}_{1}-$ Solo & $37,75 c^{\star \star \star}$ & $3,25 b$ & $18,83 \mathrm{~b}$ & $0,62 b$ \\
\hline $\mathrm{T}_{2}-$ Solo $+E \mathrm{EO}^{* * * *}$ & $55,00 \mathrm{a}$ & $4,50 \mathrm{a}$ & $19,46 \mathrm{~b}$ & $1,52 \mathrm{a}$ \\
\hline $\mathrm{T}_{3}-$ Solo $+\mathrm{CF}$ & $19,50 \mathrm{~d}$ & $2,25 \mathrm{~b}$ & $11,06 \mathrm{c}$ & $0,82 \mathrm{~b}$ \\
\hline $\mathrm{T}_{4}-$ Solo + BC & $47,50 \mathrm{~b}$ & $4,00 \mathrm{a}$ & $21,12 \mathrm{a}$ & $1,25 \mathrm{a}$ \\
\hline $\mathrm{T}_{5}-$ Solo $+\mathrm{EO}+\mathrm{CF}+\mathrm{BC}$ & $34,75 \mathrm{c}$ & $3,50 \mathrm{~b}$ & $12,49 \mathrm{c}$ & $1,35 \mathrm{a}$ \\
\hline
\end{tabular}

** Significativo ao nível de 1\%; ${ }^{* * *}$ Teste de comparação de médias de Scott e Knott ao nível de $5 \%$ de probabilidade. ${ }^{* * *}$ EO: esterco ovino, CF: cama de frango e BC: bagana de carnaúba.

O substrato solo + esterco ovino $\left(\mathrm{T}_{2}\right)$ proporcionou maior número de folhas em relação aos tratamentos $T_{1}, T_{3}, T_{4}$ e $T_{5}$ pelo teste de Scott e Knott. O tratamento $T_{3}$ foi o que apresentou menor número de folhas (Tabela 1). Paiva et al. (2011), estudaram o ENCICLOPÉDIA BIOSFERA, Centro Científico Conhecer - Jandaia-GO, v.18 n.37; p. 150 
desenvolvimento do manjericão (Ocimum basilicum L.) em diferentes substratos, e constataram que o tratamento $T_{4}$ (esterco, areia, arisco na proporção 1:1:1) em relação aos outros substratos analisados na pesquisa, isso ocorreu em decorrência da maior presença de nutrientes no substrato. Em pesquisa desenvolvida por Morais e Barbosa (2012), os autores avaliaram o efeito de diferentes adubos orgânicos (cama de frango) em associação ou não com adubo verde na produção de folhas de Ocimum selloi (alfavaquinha). Os autores observaram que não houve diferença significativa no rendimento de números de folhas da alfavaquinha nos tratamentos avaliados, reposta que ocorreu provavelmente em função da lixiviação dos nutrientes.

Em relação ao número de ramos, os substratos solo + esterco $\left(T_{2}\right)$ e o solo + bagana de carnaúba ( $\left.T_{4}\right)$ não diferiram entre si, com exceção $T_{1}$ (solo), $T_{3}$ (solo com cama de frango), e $T_{5}$ (solo, esterco ovino, cama de frango e bagana de carnaúba). $O$ número de ramos no $\mathrm{T}_{2}$ e $\mathrm{T}_{4}$ foram respectivamente igual a 4,5 e 4 (Tabela 1). Em estudo conduzido por Cunha et al. (2015) com a espécie Piper hispidum, cultivado nos substratos solo + esterco de aves e solo + casca de guaraná, os autores verificaram que o número de brotações não diferiu estatisticamente, sendo superior aos demais tratamentos analisados, que foram areia branca lavada, substrato comercial e fibra de coco.

Quanto à altura de plantas, foi possível observar que, o tratamento $T_{1}$ (solo) e $T_{2}$ (solo + esterco ovino) não diferiram estatisticamente entre si. Da mesma forma, os tratamentos $T_{5}$ (solo + esterco ovino + cama de frango + bagana de carnaúba) e $T_{3}$ (solo + cama de frango) não diferiam estatisticamente ao nível de $5 \%$ de probabilidade pelo teste de Scott e Knott. O tratamento $\mathrm{T}_{4}$ (solo + bagana de carnaúba) foi que proporcionou maior desenvolvimento da planta em altura (Tabela 1). Estudos realizados com Justicia pectoralis Jacq (chambá) por Vieira et al. (2019), utilizando três tratamentos de adubação orgânica (esterco de aves), adubação química (N:P:K), testemunha (solo sem adubação), não diferença para altura de planta 30, 60 e 90 dias de avaliação do experimento, pelo teste de Tukey ao nível de $5 \%$ de probabilidade. Ocimum selloi Benth por Costa et al. (2008), os autores reportam que altura de planta foi influenciada devido os diferentes tipos de doses de adubos orgânicos. Nhaga et al. (2019), estudando a produção de mudas do feijão bravo (Caapparis flexuosa. L) em diferentes substratos orgânicos, observaram diferença significativa entre tratamentos $T_{2}$ (solo+ esterco bovino) e o $\mathrm{T}_{3}$ (solo +esterco ovino) em relação ao $\mathrm{T}_{1}$ (solo).

Para a variável matéria seca de raiz do anador os tratamentos $T_{2}, T_{4}$ e $T_{5}$, não diferiram entre si a $5 \%$ de probabilidade, com exceção dos tratamentos $T_{1}$ e $T_{3}$ (Tabela 1). Paiva et al. (2011), em estudo realizado com manjericão, constataram que os tratamentos $\mathrm{T}_{3}$ (esterco e areia) e $\mathrm{T}_{4}$ (esterco, areia e arisco) apresentaram maior acumulo de matéria seca de raiz, em comparação aos outros substratos avaliados. Enquanto Cunha et al. (2015), estudando Piper hispidum cultivado em diferentes substratos, constataram que o tratamento solo + esterco de aves, foi superior aos demais estudados para matéria seca de raiz. Essa resposta segundo os autores pode ser atribuída à adição de matéria orgânica na forma de esterco, causando melhorias na porosidade e aeração do solo, e assim, estimulando o crescimento radicular.

Conforme o resumo da análise de variância houve diferença significativa dos substratos para as variáveis: matéria seca da parte área - MSPA $(p<0,0001)$, matéria seca total - MST $(p<0,0001)$ e relação MSR/MSPA $(p<0,0001)$, ao nível de $1 \%$ de 
probabilidade pelo teste $F$, sugerindo que os tratamentos podem ter repostas diferenciadas quando se aplicar um teste de comparação de médias (Tabela 2).

No que diz respeito à matéria seca da parte área (MSPA), o tratamento que teve menor valor foi $T_{1}$ (Solo), em relação aos tratamentos $T_{2}$ (solo + esterco ovino), $T_{3}$ (solo + cama de frango), $T_{4}$ (solo + bagana de carnaúba) e $T_{5}$ (solo + esterco ovino + cama de frango + bagana de carnaúba) na Tabela 2. Em estudo desenvolvido por Paiva et al. (2011), a matéria seca da parte área do manjericão teve melhor resposta no substrato esterco, areia e arisco $\left(\mathrm{T}_{4}\right)$.

TABELA 2. Resumo da análise variância e teste de Scott e Knott da matéria seca da parte área (MSPA), matéria seca total (MST) e relação MSR/MSPA em diferentes substratos, Redenção, Ceará, Brasil, 2019.

\begin{tabular}{|c|c|c|c|}
\hline \multirow{2}{*}{ Fonte de variação } & \multicolumn{3}{|c|}{ Quadrado Médio } \\
\hline & MSPA & MST & MSR/MSPA \\
\hline Tratamentos & $\begin{array}{c}36,261^{* *} \\
(p<0,0001)\end{array}$ & $\begin{array}{c}43,367^{* *} \\
(p<0,0001)\end{array}$ & $\begin{array}{c}0,0273^{\star *} \\
(p<0,0001)\end{array}$ \\
\hline Resíduo & 2,605 & 2,822 & 0,0016 \\
\hline Substratos & \multicolumn{3}{|c|}{ Teste de Scott e Knott } \\
\hline $\mathrm{T}_{1}$ - Solo & $1,94 b^{* * *}$ & $2,57 \mathrm{~b}$ & $0,32 \mathrm{a}$ \\
\hline $\mathrm{T}_{2}:$ Solo $+E \mathrm{O}^{* \star * *}$ & $8,67 a$ & $10,18 \mathrm{a}$ & $0,17 a$ \\
\hline $\mathrm{T}_{3}$ : Solo + CF & $8,64 a$ & $9,45 a$ & $0,10 \mathrm{~b}$ \\
\hline $\mathrm{T}_{4}:$ Solo + BC & $7,85 \mathrm{a}$ & $9,11 \mathrm{a}$ & $0,16 \mathrm{~b}$ \\
\hline $\mathrm{T}_{5}:$ Solo $+\mathrm{EO}+\mathrm{CF}+\mathrm{BC}$ & $9,20 a$ & $10,55 a$ & $0,15 b$ \\
\hline
\end{tabular}

** Significativo ao nível de 1\%; ${ }^{* * \star}$ Teste de comparação de médias de Scott e Knott ao nível de $5 \%$ de probabilidade. ${ }^{\star \star \star *}$ EO: esterco ovino, CF: cama de frango e BC: bagana de carnaúba.

$\mathrm{Na}$ variável de matéria seca total os substratos solo + esterco ovino, solo + cama de frango, solo + bagana de carnaúba e solo + esterco ovino, cama de frango + bagana de carnaúba não diferiram entre si pelo teste de comparação de médias com exceção do substrato solo (Tabela 2). Trazzi et al. (2013) avaliaram a produção de mudas de teca (Tectona grandis Linn. F.) em função de substratos de origem orgânica, constataram que nos tratamentos avaliados, a cama de frango se deferiu estatisticamente pelo teste de Tukey a $5 \%$ de probabilidade com demais.

$\mathrm{Na}$ relação entre matéria seca de raiz e matéria seca de parte área (MSR/MSPA) os menores valores foram encontrados nos substratos solo + cama de frango, solo + bagana de carnaúba e solo + esterco ovino + solo cama de frango + bagana de carnaúba em relação aos tratamentos solo e solo+ esterco ovino (Tabela 2). Em estudo conduzido por Costa et al. (2008) com Ocimum selloi Benth, variável a relação raiz: parte aérea (R: $P A)$, nas dosagens estudadas, os maiores valores foram obtidos no tratamento sem adubação testemunha, e observaram a diminuição da relação R:PA com o aumento das doses de adubo orgânico, porque, quanto maior o incremento das doses ocorre a alocação de biomassa para diferentes partes aérea da planta.

As Correlações de Pearson estimadas para número de folhas (NF), número de ramos (NR), altura de planta (Alt), matéria seca de raiz (MSR), matéria seca da parte aérea (MSPA), matéria seca total (MST) e relação entre MSR/MSPA nas plantas de anador submetido a diferentes tipos de substratos apresentaram valores entre 0,15131 e 0,87347 (Tabela 3). 
TABELA 3. Matriz de correlação entre variáveis agronômicas do anador submetidos a diferentes substratos, Redenção, Ceará, Brasil, 2019.

\begin{tabular}{|c|c|c|c|c|c|c|}
\hline Variáveis & $\mathrm{NF}^{\star \star \star}$ & NR & Alt & MSR & MSPA & MST \\
\hline NR & $\begin{array}{l}0,87347^{* *} \\
p<0,0001\end{array}$ & & & & & \\
\hline Alt & $\begin{array}{l}0,80234 \\
p<0,0001\end{array}$ & $\begin{array}{l}0,64473^{*} \\
p=0,0021\end{array}$ & & & & \\
\hline MSR & $\begin{array}{l}0,59163^{* *} \\
p=0,0060\end{array}$ & $\begin{array}{l}0,64784^{*} \\
p=0,0020\end{array}$ & $\begin{array}{l}0,16450^{n s} \\
p=0,4883\end{array}$ & & & \\
\hline MSPA & $\begin{array}{l}0,08546^{n s} \\
p=0,7202\end{array}$ & $\begin{array}{l}0,14818^{\text {ns }} \\
p=0,5330\end{array}$ & $\begin{array}{l}-0,2981 \mathrm{~ns} \\
\mathrm{p}=0,2018\end{array}$ & $\begin{array}{l}0,58368 \\
p=0,0069\end{array}$ & & \\
\hline MST & $\begin{array}{l}0,15131^{n s} \\
p=0,5243\end{array}$ & $\begin{array}{l}0,21611^{n s} \\
p=0,3601\end{array}$ & $\begin{array}{c}-0,2552^{n s} \\
p=, 2775\end{array}$ & $\begin{array}{l}0,66144 \\
p=, 0015\end{array}$ & $\begin{array}{l}0,99506^{* *} \\
p<0,0001\end{array}$ & \\
\hline MSR/MSPA & $\begin{array}{l}0,24324^{n s} \\
p=0,3014\end{array}$ & $\begin{array}{l}0,16579^{n s} \\
p=0,4848\end{array}$ & $\begin{array}{l}0,447697^{*} \\
p=0,0478\end{array}$ & $\begin{array}{l}-0,1667^{n s} \\
p=0,4824\end{array}$ & $\begin{array}{l}-0,8363^{\star *} \\
p<0,0001\end{array}$ & $\begin{array}{l}-0,7928 \\
p<0,0001\end{array}$ \\
\hline
\end{tabular}

Não significativo $\left({ }^{\mathrm{NS}}\right)$, significativo ao nível de $5 \%\left({ }^{*}\right)$ e significativo ao nível de $1 \%\left(^{* *}\right)$ pelo teste $t .{ }^{* * *}$ NF: número de folhas, NR: número de ramos, Alt: altura da planta, MSR: matéria seca de raiz; MSPA: matéria seca de parte área, MST: matéria seca total e relação MSR/MSPA.

As combinações NF $\times$ NR ( $r=0,87347)$, NF $\times$ Alt $(r=0,80234)$, NF $\times$ MSR ( $r=$ $0,59163)$ resultaram em valores significativos pelo teste $t(p<0,01)$, expressando que número de folhas é influenciado positivamente pelas variáveis número de ramos, altura da planta e pela matéria seca de raiz. Ao passo que as correlações entre os pares NF $x$ MSPA ( $r=00,08546)$, NF x MST $(r=0,15131)$ e NF $\times$ MSR/MSPA $(r=0,24324)$ não apresentaram respostas significativas.

\section{CONCLUSÕES}

Os substratos solo + esterco ovino $\left(T_{2}\right)$ e solo + bagana de carnaúba $\left(T_{4}\right)$ proporcionaram melhor propagação das plantas de anador.

Os substratos solo $\left(T_{1}\right)$, solo + cama de frango $\left(T_{3}\right)$ e solo + esterco ovino + cama de frango+ bagana de carnaúba $\left(T_{5}\right)$ não são recomendados para o cultivo do anador.

\section{REFERÊNCIAS}

BARBOSA, J. C.; MALDONADO JUNIOR. W. AgroEstat- Sistema para Análises Estatísticas de Ensaios Agronômicos. Versão 1.1.0.712, Jabotical: FCAV/UNESP, 2015, 396p.

BERACUHY, J. G. V.; FURTADO, D. A.; FRANCISCO, P. R. M.; LIMA, J. L. S.; PEREIRA, J. P. G. Plantas medicinais de uso comum no Nordeste do Brasil. Campina Grande: EDUFCG, 2016, 205p.

CARVALHO, M.; CAIXETA, G. A. B.; LIMA, A. R. S.; TEOFILO, M. N. G.; CRUVINEL, W. M.; GOMES, C. M.; FLEURY, L. F. F;. PAULA, J. A. M. AMARAL, V. C. S. Assessing the safety of using the dry extract of Justicia pectoralis Jacq. (Acanthaceae) during pregnancy of Wistar rats. Journal of Ethnopharmacology, v.268, p.1-7, 2021. DOI: https://doi.org/10.1016/j.jep.2020.113618. 
COSTA, L. C. B.; PINTO, J. E. B. P.; CASTRO, E. M. ; BERTOLUCCI, S. K. V.; CORRÊA, R. M.; REIS, E. S.; ALVES, P. B.; NICULAU, E. S.Tipos e doses de adubação orgânica no crescimento, no rendimento e na composição química do óleo essencial de elixir paregórico. Ciência Rural, Santa Maria, v.38, n.8, p.2173-2180, 2008. Disponível em: <http://www.scielo.br/pdf/cr/v38n8/a13v38n8.pdf>.

CUNHA, A. L. B.; CHAVES, F. C. M.; BATISTA, A. C.; HIDALGO, A. F. Propagação vegetativa de estacas de Piper hispidum Sw. em diferentes substratos. Revista Brasileira Planta Medicinais, Campinas, v.17, n.4, suplemento. I, p.685-692, 2015. Disponível em: <http://www.scielo.br/pdf/rbpm/v17n4s1/1516-0572-rbpm-17-4-s10685.pdf>.

GOELZER, A.; SILVA, O. B.; TORALES, E. P.; SANTOS, C. C.; VIEIRA, M. C. Tipos de recipientes na propagação por estaquia de três espécies medicinais. In: A produção do Conhecimento nas Ciências Agrárias e Ambientais. Ponta Grossa: Atena, 2019, p.157167.

HARTMANN, H. T.; KESTER, D. E.; DAVIES Jr, F. T.; GENEVE, R. L.; WILSON, S. E. Plant propagation: principles and practices. 9. ed. New Jersey: Prentice Hall, 2018. 1024p.

IPCE - Instituto de Pesquisa e Estratégia Econômica do Ceara. Perfil básico municipal - Redenção. 2017a. Disponível em: <https://www.ipece.ce.gov.br/wpcontent/uploads/sites/45/2018/09/Redenca2017.pdf>.

IPCE - Instituto de Pesquisa e Estratégia Econômica do Ceara. Perfil básico municipal - Fortaleza, Ceará. 2017b. Disponível em: <https://www.ipece.ce.gov.br/wpcontent/uploads/sites/45/2018/09/Fortaleza_2017.pdf>.

LEAL, L. K. A. M.; SILVA, A. H.; VIANA, G. S. B. Justicia pectoralis, a coumarin medicinal plant have potential for the development of antiasthmatic drugs? Revista Brasileira de Farmacognosia. v.27, p.794-802, 2017. DOI: https://doi.org/10.1016/j.bjp.2017.09.005.

LAMEIRA, O. A.; PINTO, J. E. B. P. Plantas Medicinais: do cultivo, manipulação e uso à recomendação popular. Editora EMBRAPA: Belém, 2008, p.19-26.

LORENZI, H.; MATOS, F. J. A. Plantas Medicinais no Brasil: nativas e exoticas. $2^{a}$ ed, Ed. Plantarum de Estudos da Flora Ltda., Nova Odessa, SP., 2008, 512p.

MASIERO, M. A.; ORIVES, K. G. R.; CRUZ, L. C.; AMÂNCIO, J. S.; FELICETI, M. L.; VIANA, C. M. S. S.; LIMA, D. M. Uso de substratos na estaquia de astrapéia (Dombeya wallichii L.). Revista Cultura Agronômica, Ilha solteira, v.28, n.3, p.241-253, 2019. DOI: https://doi.org/10.32929/2446-8355.2019v28n3p241-253.

MORAIS, L. A. S.; BARBOSA, A. G. Influência da adubação verde e diferentes adubos orgânicos na produção de fitomassa aérea de atroveran (Ocimum selloi Benth.). Revista ENCICLOPÉDIA BIOSFERA, Centro Científico Conhecer - Jandaia-GO, v.18 n.37; p. 154 2021 
Brasileira de Plantas Medicinal, Botucatu, v.14, número especial., p.246-249, 2012. Disponível em:<http://www.scielo.br/pdf/rbpm/v14nspe/20.pdf>.

NHAGA, A. O.; PINTO, C. M.; SALLES, M. G. F.; PINTO, O. R. O.; NETO, A. M. V. Produção de Mudas do Feijão Bravo em Diferentes Substratos Orgânicos. Enciclopédia Biosfera, Centro Cientifico Conhecer - Goiânia, v.16 n.29; p.1, 2019. DOI: 10.18677/EnciBio_2019A108

PAIVA, E. P.; MAIA, S.S.S.; CUNHA, S. M.; COELHO, M. F.B.; SILVA, F.N. Composição do substrato para o desenvolvimento de mudas de manjericão (Ocimum basilicum L.). Revista Caatinga, Mossoró, v. 24, n. 4, p. 62-67, 2011. Disponível em: <https://periodicos.ufersa.edu.br/index.php/caatinga/article/view/2083/pdf>.

PRADO, M. A. S. A.; MATSUOK, J. T.; GIOTTO, A. C. A importância das farmácias vivas no âmbito da produção dos medicamentos Fitoterápicos. Revista Iniciação Científica e Extenção, v.1, n.1, p.32-37, 2018. Disponível em: <https://revistasfacesa.senaaires.com.br/index.php/iniciacao-cientifica/article/view/45>.

SILVA, A. M.; PAES, L. W. C.; MARTINAZZO, A. P.; SOUZA, P. P.; MARTINS, F. A. C. B. Plantas medicinais como recurso terapêutico nos bairros Siderópolis e Santa Rita do Zarur em Volta Redonda/RJ, Brazilian Journal of Development, Curitiba, v.7, n.2, p.11997-12014, 2021. DOI: 10.34117/bjdv7n2-023

VIEIRA, R. M.; VIEIRA, A. S.; REZENDE, C. F. A.; PEIXOTO, J. C. Crescimento, acúmulo de nutriente e prospecção fitoquímica da justicia pectoralis jacq em função do tipo de adubação. Revista Brasileira de Agropecuária Sustentável (RBAS), v.9, n.4, p.27-33, 2019. DOI: https://doi.org/10.21206/rbas.v9i04.8601

VIEIRA, A. C. M.; ANDRADE, S. R.; SEIXAS, I. M. V.; MEDEIROS, T. K. C.; CARNEIRO, L. S. M. Manual sobre uso racional de plantas medicinais. 1. ed., Rio de Janeiro: $\quad$ Cerceau, $2016.2175 \mathrm{p} . \quad$ Disponível: <https://www.researchgate.net/publication/311736167_Manual_Sobre_Uso_Racional_d e_Plantas_Medicinais__-_Volume1>.

TRAZZI, P. A.; CALDEIRA, M. V. W.; PASSOS, R. R.; GONÇALVES, E. O. Substratos de Origem Orgânica para Produção de Mudas de teca (Tectona grandis Linn. F.). Ciência Florestal, Santa Maria, v. 23, n. 3, p. 401-409, 2013. DOI: https://doi.org/10.5902/1980509810551. 